\title{
Enhancing Disaster Preparedness of Specialty Nurses on a National Scale
}

\section{Authors:}

1) Marion L. Mitchell ${ }^{1,2}$, PhD, RN, FACCCN.

Joint Appointment Griffith University and Princess Alexandra Hospital. Australia.

2) Loretta McKinnon ${ }^{1}, \mathrm{PhD}, \mathrm{BA}, \mathrm{BSc}$

$\mathrm{PhD}$ prepared senior research fellow

3) Leanne M Aitken ${ }^{1,2,3}$, PhD, RN, FACN, FAAN.

Joint Appointment Griffith University and Princess Alexandra Hospital, Australia.

4) Sarah Weber ${ }^{2}$, BN, Grad Cert (Emergency and Disaster Management)

Clinical Nurse Consultant - Counter Disaster Department.

5) Sean Birgin ${ }^{2}$, BN

Director of Nursing - Surgery, Princess Alexandrea Hospital, Australia.

6) Sharon Sykes ${ }^{4}$, BN

Assistant Director of Nursing, Intensive care Unit, Royal Darwin Hospital, Darwin, Australia.

\section{Authors' Affiliations:}

${ }^{1}$ School of Nursing and Midwifery and Centre for Health Practice Innovation, Menzies Health Institute Queensland, Griffith University, Brisbane, Australia.

${ }^{2}$ Intensive Care Unit, Princess Alexandra Hospital, Brisbane, Australia.

${ }^{3}$ School of Health Sciences, City University London, UK.

${ }^{4}$ Royal Darwin Hospital, Darwin, Australia.

\section{Correspondence:}

Marion Mitchell, Intensive Care Unit, Princess Alexandra Hospital, Ipswich Road, Woolloongabba, 4120, Brisbane, Australia

Email: marion.mitchell@griffith.edu.au,

Fax: +6173176 7356,

Phone: +61 73176 7772). 


\begin{abstract}
Purpose

The number of disasters has increased by $30 \%$ worldwide in the past 30 years. Nurses constitute the largest clinical group within a hospital and their ability to respond to disasters is crucial to the provision of quality patient care. The aim of this study was to evaluate a fouryear disaster preparedness partnership between two tertiary hospitals from the perspective of specialist nurses, senior clinical managers and executive staff. The national disaster response centre was situated in one hospital and the other hospital was located 3,500 km away.

Design
\end{abstract}

The intervention involved selected nurses working at the partner hospital to enable familiarisation with policies, procedures and layout in the event of a request for back-up in the setting of a national disaster. A mixed-methods design was used to elicit the strengths and limitations of the partnership. Data were collected through surveys, in-depth interviews and focus groups.

\title{
Findings
}

Sixty-seven participants provided evaluations including 38 nurses from the Disaster Support Response Team; 17 clinical management nurses and 10 executive staff. Improvements in some aspects of communication were recommended. The successful recruitment of highly skilled and committed nurses was a strength. A disaster exercise resulted in $79 \%$ of nurses contacted, able and willing to go immediately to the partner hospital for up to 14 days.

\section{Limitations}

During the four year partnership, no actual disaster occurred that required support. This limited the ability to fully trial the partnership in an authentic manner. The disaster exercise, although helpful in trialing the processes and assessing nurse availability, has some limitations. Financial evaluation was beyond the scope of the project. 


\section{Value}

This innovative partnership successfully prepared specialist nurses from geographically distant hospitals to provide 'back-fill' to support a disaster response. This together with a willingness to be deployed enhanced Australia's capacity in the event of a disaster. 


\section{Introduction}

The number of reported disasters has increased by around $30 \%$ worldwide in the past 30 years (Centre for Research on the Epidemiology of Disasters, 2009). It has been predicted that by 2015 the number of people affected yearly by climate-related disasters alone will have risen to 375 million (Department for International Development, 2011). Additionally, as urbanisation increases in under-developed countries, infrastructure can be poorly planned and when disaster strikes the consequences are catastrophic (Albrito 2012). Disasters can quickly overwhelm local services necessitating assistance from external sources (Redmond 2005, Djalali, Ingrassia et al. 2014). Within Australia, most recently in April and also in March, 2015, medical disaster teams have been sent to assist with the earthquake in Nepal and to Vanuatu to provide immediate care following the devastating Category 5 cyclone, "Pam". Similarly, patient care disaster support was provided to the Philippines following typhoon Haiyan and locally to people affected by cyclone Yasi in 2011 in north Queensland.

Importantly, disaster events are not predictable as they can include acts of terrorism, climate variability and change, animal and human diseases and risk of global epidemic (International Federation of Red Cross and Red Crescent Societies, 2011; Lesus, 2000). It is crucial that nurses, who constitute the largest group of health care providers, are able to leave their usual place of employment to form disaster response teams (Pang et al, 2009; Gorlin and Hick, 2013). Preparedness activities facilitate an ability to meet the demands of a large incident in a controlled and optimal manner. However, it is cautioned that nurses willingness to come to work during a disaster, depends on a combination of both personal and work related factors (Arbon, et al, 2013). It is however, recommended that education is provided to all student nurses within their degree programme (Jennings-Sanders, Frisch and Wing, 2005), however, 
interdisciplinary education is desirable as it promotes team work (Duarte \& Haynes, 2006). Education may be delivered in a number of ways including different modes of simulation which can be tailored to suit the availability of resources and desired educational outcomes (Pattillo, 2006). Both work related skills and adequate resources were found to be crucial in a review of 16 research papers (Chapman and Arbon, 2008). Gorlin and Hick (2013) report that after the Minneapolis bridge collapse in 2007 the co-ordinated disaster response was successful thanks to disaster planning which included drills co-ordinating responses across jurisdictions and departments. In this paper a four-year disaster preparedness project between two geographically distant tertiary Australian hospitals is reported.

Australia has a National Critical Care Trauma Response Centre (NCCTRC) which is situated within the Royal Darwin Hospital (RDH) in the Northern Territory. Through a tendering process, Princess Alexandra Hospital (PAH) in Brisbane $(3,500 \mathrm{~km}$ away) was selected to partner with the RDH to provide skilled staff to help meet the nursing needs of a disaster. Specifically, the PAH provided senior nurses to work within the intensive care unit (ICU), emergency department (ED), perioperative (PACU), and operating theatre (OT) of the RDH with the plan to relieve RDH nurses who would be deployed to the disaster event. That is, the PAH nurses were to provide a 'back-fill' function at the $\mathrm{RDH}$ and are termed in this paper Disaster Support Response Team (DSRT) members. This partnership was evaluated six months after its commencement and has been reported previously (Mitchell et al., 2014). The current study reports on the entire four years of the project during which time $104 \mathrm{PAH}$ nurses went to $\mathrm{RDH}$ to work in their respective unit for familiarisation and orientation purposes. 
Experienced senior PAH nurses were accepted into the DSRT if they met the criteria of an ability to demonstrate clinical expertise including leadership qualities and an ability to work effectively in stressful situations. Each specialist nurse went to work at the RDH for a twoweek period. A second rotation of one week occurred approximately 12 months later to consolidate learning and maintain relationships with RDH colleagues. The purpose of this study was to evaluate the partnership from the perspective of key stakeholders including specialist nurses, senior clinical managers and executive staff to identify both positive aspects and areas for improvement.

\section{Methods}

Qualitative and quantitative methods were combined to explore staff perceptions of the partnership between the PAH, RDH and NCCTRC and provide a descriptive analysis of staff responses. Mixed methods are an effective means of capturing perspectives from multiple stakeholders (Teddie and Tashakkori, 2013) and promote a comprehensive understanding of the subject matter under study (Annells and Whitehead, 2007; Creswell, 2009; Jones and Bugge, 2006).

Sites

The NCCTRC is located within the RDH and has the capacity to respond to disasters both within and outside Australia. Both the PAH and RDH provide a broad range of health care and are large, metropolitan, tertiary teaching hospitals. The RDH provides speciality services to the Darwin populace. In addition, $\mathrm{RDH}$ serves as a referral centre to northern Western Australia, the Northern Territory, and South-East Asia. Cumulatively the RDH serves approximately 150,000 people. The PAH while being 3,500 kilometres from Darwin is one of the closest metropolitan areas and specialist referral centres for the RDH. 


\section{Sample}

Feedback was sought from three levels of staff (from both hospitals). These were: PAH Disaster Support Response Team (DSRT) members (highly skilled nurses); clinical management nurses (responsible for managing the DSRT, clinical management nurses included: educators, Nurse Unit Managers, Clinical Nurse Consultants, Clinical Facilitators, and Directors of Nursing); and executive staff (responsible for the design, inception, and ongoing management of the partnership). All potential participants from these three areas were invited to provide feedback by either survey and focus group or interviews. Recruitment strategies included emailing all potential participants via their staff email with a follow-up email or telephone communication to determine the timing for focus groups/interviews. Interviews were offered to executive staff and to participants who were unable to attend a focus group but wished to provide feedback.

\section{Data collection instruments}

\section{$\underline{\text { Surveys }}$}

Separate surveys were issued to all clinical management nurses and all members of the DSRT. These surveys have been used previously (Mitchell et al., 2014) and included structured-response and open-ended items. The survey issued to clinical management nurses included 15 items covering the impact of rotations on: staff rostering, skill mix, staff workload, work culture, and the overall value of the partnership. The DSRT survey had 38 items that collectively assessed their perceptions of preparedness for the RDH rotations and prospective deployment, confidence in knowledge and skills, perceptions of the work environment in $\mathrm{RDH}$, sources of professional fulfilment and preparedness to leave their family if called. 


\section{$\underline{\text { In-depth interviews/focus groups }}$}

All three areas of staff were asked to outline what they felt were the positive and negative aspects of the partnership and to raise any suggestions for improvement. Staff at the executive level were asked some unique questions about the objectives of the partnership and whether these were met, namely whether DSRT nurses were sufficiently prepared and available when needed. Staff at the clinical-management level were asked questions regarding rostering and changes in skill mix in their unit, and were also asked to articulate challenges or benefits arising from the partnership. DSRT members were asked about the adequacy of preparations for their potential deployment to Darwin and also about arising benefits and issues concerning their involvement in the partnership.

\section{Data collection}

Data collection was undertaken by the first and second authors who are PAH researchers with no affiliations with the DSRT, NCCTRC or RDH. Data collection occurred from January to June 2014. All participants were invited to participate in an in-depth interview or focus group. Interviews were recorded, transcribed verbatim and emailed to participants for verification of their accuracy and completeness.

\section{Data analysis}

The quantitative data were analysed using SPSS (Version 19.0. Armonk, New York: IBM Corp.). The qualitative data (derived from open-ended survey questions, focus groups and indepth interviews) were read and reread and assessed independently by the first and second authors. Thematic analysis was conducted and codes were derived by each researcher based on identified meanings, topics, or keywords and were then reconsidered collaboratively to 
produce a final, cohesive set with themes and sub-themes. Further refinement and decisions on final themes were in collaboration with the other authors.

\section{Ethical considerations}

Ethical approval was obtained at both hospitals. Participation was voluntary. Return of completed surveys deemed consent. Focus group/interview participants provided written informed consent.

\section{Results}

Sixty-seven participants provided feedback: DSRT members $(n=38)$; clinical management nurses (RDH n= 9, PAH n=10); and executive staff (NCCTRC $n=5$, RDH n=1, PAH n=4). Among the DSRT, participation rates for the survey were $48 \%$ (38/79). Twenty-four of the 79 participated in focus groups or interviews that took from 30- 60 minutes. All specialty areas were well represented. Emails were sent to 79 listed DSRT members, however since some were on leave or working in another area during the data collection period it was not possible to determine how many staff received the invitational email. Participation rates were high (>89\%) among clinical management and executive staff. Overall a representative sample was achieved.

DSRT participants were predominantly women $(76.3 \%, \mathrm{n}=29)$ and the majority were aged 25 to 34 years $(52.6 \%, \mathrm{n}=20)$. Approximately half had completed a single rotation to Darwin $(47.4 \%, \mathrm{n}=18)$, a quarter had completed two $(26.3 \%, \mathrm{n}=10)$, and ten $(26.4 \%)$ had completed three or more rotations. Representation from each of the four work areas was similar (Table 1).

$* *$ table $1 * *$ 
Data from all three participant groups were combined to ascertain the features of the partnership perceived as working well and those areas that required improvement. Data analysis revealed four themes that included: 1) nurse characteristics, 2) preparation of nurses, 3) procedures of the partnership and 4) partnership outcomes.

Theme 1) Nurse Characteristics

A number of characteristics of the DSRT members were identified by all participants as important for success included being highly skilled, motivated, willing, committed, and available.

Executive staff and clinical management nurses in Darwin and in Brisbane, noted that DSRT members were highly skilled which was considered very important. PAH clinical management nurses (CMN) spoke of how DSRT positions were highly sought after and were only offered to the most senior, competent and experienced nurses. DSRT members also spoke of the competitiveness of gaining a place in the partnership.

"...the people that were on the rotations... they were all senior staff, and we did that obviously because we thought that they'd [RDH]get the most benefit, and we'd get the most benefit back by sending our senior staff." [PAH CMN ED, 11]

CMN at the RDH described how the high skill level of the DSRT members made them highly valuable as they were able to act as senior staff members in a variety of nursing contexts.

"Due to the skills, knowledge, and expertise of PAH staff they were able to be allocated to a theatre straight away, they could be used straight away". [RDH CMN OT, 3]

Executive staff (Ex) and CMN at the RDH elaborated to say that the DSRT being highly skilled was a boost for morale and gave those responsible for rostering staff great peace of mind. 
"With our hospital supervisors, when I go home at night and I ask "how's the hospital?", they are very much, "[ Name], don't worry. We've got PAH [staff]". Okay, no worries, done. So it was that easy, [it eased] that burden". [RDH Ex, 6]

The DSRT members displayed motivation and engagement. CMN from the PAH and RDH commented on the high levels of enthusiasm and commitment displayed among the DSRT. When speaking about adaptability and willingness of the DSRT members, CMN often commented on the nurses' motivation, flexibility and strong desire to contribute.

"They wanted to get stuck in there, [nurse's names] in particular would just throw themselves in. They loved it, they loved how busy it was". [RDH CMN OT, 2]

High levels of motivation were evident which may have been aligned to the difficulty of being selected for the DSRT. One nurses described waiting a number of years to obtain a place.

"I saw it being an exclusive sort of thing, something that I really wanted to be a part of. You know you see senior nurses doing it and I was like, I really want to be a part of that. Then to finally get a spot, I was so happy..." [PAH DSRT ICU, 26]

Many DSRT members described preparations that they had made with their family to ensure that they could be deployed. One nurse described a goal that may have been common to others, that she was committed to keeping herself physically fit so that she would be of the utmost value to the RDH/NCCTRC should she be deployed.

Both CMN and DSRT members across units commented on high rates of retention in the program with members generally only withdrawing if they left the hospital or unit or due to family obligations (e.g. expecting a baby). Many staff re-iterated the seriousness of their commitment to the program, explaining that they would absolutely have withdrawn 
themselves had their circumstances changed rendering them no longer able to be a reliable team member.

The ability to recruit highly skilled and committed nurses was dependent upon the selection procedures employed by the PAH. Although not called upon to do so for an actual emergency, a disaster exercise was undertaken and revealed high numbers of staff willing to go to RDH. In total, $79 \%(n=37)$ of staff who were contacted $(n=47)$ were available for immediate deployment for up to 14 days. This response rate was more than adequate and represents a favourable response to the call for deployment in the exercise.

\section{Theme 2) Preparation of nurses}

CMN at both sites and DSRT members emphasised the importance of suitable preparation of DSRT members to allow them to be highly functioning staff members in the event of deployment to RDH. While three quarters of the DSRT indicated that they were 'well' prepared $(n=24)$ or 'very well' prepared $(n=4)$ for deployed to the $R D H$, not all team members felt completely ready. Such team members indicated that they felt 'somewhat' prepared $(n=9)$, with one staff member who had one, two-week period in RDH indicating that they were 'not well' prepared $(n=1)$.

The following sections detail crucial aspects of preparation identified by participants including: competence and knowledge of expectations; the importance of rotations, specific information and education needs and family preparedness. 
The DSRT members were asked a number of questions concerning self-assessment of their confidence in their knowledge and skills as a DSRT member. As shown in Table 2, almost all staff either 'strongly agreed' or 'agreed' that they had the required knowledge and skills.

$* *$ table $2 * *$

On-site clinical rotations were important. DSRT members commented that the initial twoweek period was an appropriate length of time, and that subsequent visits of one-week were also appropriate for re-familiarisation.

Educational needs were highlighted by DSRT members and ranged from being able to operate equipment to cultural matters. Equipment in particular was noted as an important reason for why rotations were necessary rather than virtual learning. Equipment information needs appeared particularly relevant for those in the ICU.

"It's not the big stuff, because the big stuff doesn't change from one
unit to another... It's the little stuff, but you can't do the big stuff
without the little stuff. So it's knowing where to find equipment in the
storeroom. It's knowing that you've got a password to use the blood
gas analyser. "' [DSRT ICU, 26]

Across areas, DSRT members commented on their lack of familiarity with paediatric patients. Some DSRT members and CMN commented that they felt confident that DSRT members would not be left alone with children if they were not confident to care for them.

"Some were really keen, others were terrified. But all of them had a go and I think they were generally well supported when they were in with the 'kids'... and I don't think any of them were pushed into it'. [PAH CMN OT, 15]

Many DSRT members acknowledged cultural differences in the patient base between Darwin and Brisbane.

"We don't have the ATSI [Aboriginal and Torres Strait Islander patient] dealings that they have up there..." [DSRT ICU, 26] 
"Working with all those different Aboriginal communities. They speak in their dialect and [we learnt] how to work with them and their community. Just because they're not in the bed doesn't mean they've left, they're just outside warming up [from the cool airconditioning].[DSRT ED, 22]"

A number of DSRT members commented that the cultural training that they received was both excellent and essential. The quantitative data (see Table 3) were consistent with the qualitative data, in showing that most DSRT members felt well prepared to care for Aboriginal and Torres Strait Islander patients and their families, and could locate the relevant resources to do this.

** table $3 * *$

However, despite confidence in being prepared displayed in both the quantitative and qualitative findings, DSRT members also commented that more educational opportunities with regard to cultural matters would have been welcome.

"I don't think you could ever get enough training in that to be quite honest. Because there's so many different cultures within that culture, and that's where the liaison person was the intermediary. " [DSRT ICU, 26]

DSRT members also expressed their preference to receive cultural sensitivity training in

Darwin, so that they could apply what they were learning in context.

"Yeah, like even little things like lying in the bed and covering their head, things like that. I mean it makes no sense if you were sitting in a classroom ... It lacks context, I mean when you see it with your patient you think oh, okay cool, I understand what's going on there now".

[DSRT ICU, 26]

Many DSRT members had spouses and families to consider. DSRT members generally reported that the rotations had 'no impact' $(45 \%, \mathrm{n}=17)$ or 'some impact' $(47 \%, \mathrm{n}=18)$ on themselves and their families, with fewer staff $(8 \%, n=3)$ reporting a greater impact. There 
were no consistent trends in terms of family status (e.g. partnered or not, children or not) and perceived impact of rotations.

Theme 3) Essential partnership procedures

There were a number of operational matters that influenced the success of the partnership including: orientation, team work and logistics such as accommodation.

When DSRT members arrived in Darwin generally they were given an orientation to the NCCTRC, RDH, and their specific work unit. The appraisal of the adequacy of the orientation varied. Some DSRT members noted that their unit (e.g. ICU, ED, PACU or OT) did not know that they were coming and this greatly affected the DSRT, both in a practical sense and in terms of morale. Not knowing that they were coming meant that no time had been set aside to show DSRT members around and administrative tasks such as receiving a name badge, and computing access were delayed. Some staff members reported receiving a more thorough introduction to their unit than others. This appeared to vary according to different timing and circumstances as opposed to differing across hospital units.

"When I went up there, we were very short-staffed and there was no supernumerary or someone guiding us around. So it was like, there you go, you've never done this before, well learn while you're doing, sort of thing. So I think you had to learn fairly quickly when you were up there." [DSRT OT, 18]

Many DSRT members and CMN at both sites, commented on the good relations between PAH and RDH staff. DSRT members noted specifically that they tried not to 'step on anyone's toes' and that while they were in Darwin they made a concerted effort not to present themselves as 'knowing best' because they were from a 'big city' hospital.

"It's Darwin, that's how they do it. We're not here to change them and you've just got to [do it that way] - and that's what they would tell 
the next lot of people that were going up... The idea is that you're going to relieve them and you're not there to take over and change their practice". [PAH CMN OT, 15]

As shown in Table 4, most DSRT members felt that they had the skills and knowledge to develop effective working relationships and felt accepted by their co-workers in Darwin. An analysis of the five team members who disagreed that they were accepted by the RDH multidisciplinary team showed no trend in terms of their work area (e.g. ICU, OT, PACU or ED).

$* *$ table $4 * *$

Accommodation and transport was noted by most DSRT members to be adequate. Some changes occurred over the course of the partnership with cheaper accommodation options being trialled. DSRT members and CMN noted the importance of supplying accommodation that was adequate in terms of safety and hygiene. Below are some contrasting sentiments on the accommodation.

"We stayed at the (accommodation named) and it was excellent". And [in relation to the trialled cheaper housing option] "It was yeah, yes it was [substandard], and I've been in the army. We didn't have a number of very simple items that you'd find in the average motel style accommodations, so we bought those". [DSRT ICU, 26]

There was some impact on sending staff to Darwin for rotations. Responses among PAH and RDH CMN varied with staff indicating that the partnership either had 'some', 'moderate', or a 'large' impact on staff rostering (Table 5) .

$* *$ table $5 * *$ 
Careful planning was required prior to the rotations to ensure sufficient staff numbers were retained at the $\mathrm{PAH}$, however this did not always occur.

“... because anaesthetic nurses work one-on-one ...in my role I had to close a theatre because we didn't have enough anaesthetic staff and trying to steal nurses for elsewhere, it became a bit tense at times". [PAH CMN OT, 15]

Those respondents from Darwin who indicated that the rotations had a large impact, qualified that the PAH partnership was of great value to help overcome staff shortages, in other words it was a large positive impact.

"The staffing [was] impacted positively...it was a large impact because I know when we had deficits, we knew the PAH staff were coming, it was like, great, we've got senior staff on their way." [CMN RDH OT, 3]

Communication was noted as an area for improvement in the partnership by a number of participants. RDH CMN indicated that they were not sure who they should be communicating with at the PAH and there were some instances where PAH staff were uncertain about the line-of-command and who they should report to while in Darwin.

Some aspects of communication were noted to have improved over time. The recruitment of a nurse manager dedicated to the partnership was seen as pivotal in enhancing communication. In the early stages of the partnership there were no PAH clinical facilitators involved. Once clinical facilitators were recruited, they were described as playing a vital role in supporting communication between the nurse manager and the two hospitals' staff.

"The nurse manager was trying to cover everybody and deal with backwards and forward with Darwin and all that sort of stuff. ...the clinical facilitator wasn't just about the paperwork. She was here for the staff and wanted their feedback, wanted to sort the issues out". [PAH CMN OT, 13]

Theme 4) Overall Partnership Outcomes 
The main purpose of the partnership was to provide adequate numbers of suitably qualified nurses to RDH upon demand in a disaster to provide 'back-fill'. This goal was met in terms of the selection of highly skilled and committed staff who perceived that they received adequate preparation to be deployed. The disaster exercise call for deployment indicated that a high proportion of team members (79\%) were available and willing to be deployed. Staff at the NCCTRC and RDH confirmed that they felt confident that the PAH could support them in times of disaster.

"When we needed the PAH in terms of disaster, it was easy. I could ring [Director of NCCTRC]. He contacted the counterparty at PAH... and then things happen". [RDH Ex, 6]

Across stakeholder groups, although suggestions for improvement to the partnership were noted it was also widely remarked that the partnership had improved over time with many issues being resolved.

"Some of our staff had never travelled before. So I know over the
course of the four years we put better screening tools in place for our
staff. There are a whole lot of other things that we also factored in as
we progressed through the four years. So I think that may have been
an opportunity for us but it was certainly a barrier for us at the
beginning because we didn't recognise that some of our staff had
never been away from home". [PAH Ex, 28]

In addition to the core objective of the partnership being met, participants outlined many ancillary benefits of the collaboration that are detailed in the sections below.

Both PAH CMN and DSRT members commented on the opportunities that the DSRT had to encounter new experiences. As noted earlier, PAH DSRT members cared for Aboriginal and Torres Strait Islander and paediatric patients. Exposure to tropical diseases was also noted as a valuable learning experience.

"I think from a whole clinical point of view that our staff had an opportunity to see disease processes that they wouldn't have seen in 
Brisbane. So, definitely... many of them wouldn't have experienced tropical disease”. [PAH Ex, 28]

Staff from both the PAH and RDH expressed that being involved in the partnership allowed them valuable exposure to other hospital's practices and procedures that prompted them to question what they had been used to.

"I think another benefit for us as a team from ICU is that this unit has a very low turnover of nursing staff... We're very protocol driven in this unit, we have a clinical guideline for everything. So it's really good to go and work in another unit, and see how they do the same thing, but differently. It makes you stop and think about what we do and how we do it, and could we do it better". [PAH DSRT ICU, 25]

DSRT members shared the experiences and knowledge that they gained with their colleagues when they got home. In addition to learning while at the RDH, DSRT members also provided learning opportunities to RDH staff through mentorship, in-services and by providing information about equipment, procedures and protocols.

"If we were not up to date on a certain area they would share information from the PAH with us. For example, they provided us information on endoscopes as we were trying to come up to Australian standards. This is one area of the relationship that could improve in future though as we didn't always get the information we requested, we believe due to hospital protocols on sharing information". [RDH CNS OT, 3]

Some team members emphasised that the information sharing was an ongoing legacy of the partnership.

"I was down here in charge [at PAH ICU] and we had a patient on the oscillator ventilator. It kept blowing the bellows and that's a very unusual thing. So we could not get hold of the rep [company representative]. So because I had been to Darwin I called Darwin, because they use it a lot. I called them up and I knew the nurse who was in charge, we chatted for a minute. I said, hey you know I've got this problem with the oscillator, listen to this, held the phone up to it and she diagnosed the problem straight away and told me how to fix it. That was at about $11 \mathrm{pm}$ - we did what she said around midnight and it's hard to get support at $11 o^{\prime}$ clock at night. So that was a great phone call, she's very helpful'”. [DSRT ICU, 25] 
Executive staff at all partner entities acknowledged information sharing as a particularly useful benefit of the partnership.

"One of the great successes of the PAH intervention, was to bring PAH staff across here to complement and enhance teaching to give us a new perspective, and from a nursing perspective that happened spectacularly”. [NCCTRC Ex, 1]

This knowledge sharing had the added advantage of enhancing disaster preparedness planning within the PAH. DSRT members commented that their unit's disaster plans have been updated and are now in-line with best practice following consultations with RDH colleagues.

As noted previously, interpersonal relationships between the DSRT and RDH staff were generally good. In some cases lasting collegial relationships and friendships were formed. RDH staff expressed their warm feelings towards the PAH staff and said that they looked forward to having them there.

PAH staff spoke of the benefits of getting to know local staff in other units through bonding while away. The resulting contact led to better understand the needs of different work areas, facilitating greater efficiency when working together. Executive and CMN spoke of the organisational relationships developed. Across staff levels, many study participants spoke of the continued collegial relationships and information sharing to be a wonderful and ongoing benefit of the partnership.

"I think from my point of view it [the partnership] allowed me to develop a strong relationship with their Director of Nursing at the $R D H$ and that's something that we certainly didn't have before the partnership, and equally with the Executive Director of the NCCTRC. We pick up the phone and talk to each other now. So that's a strengthI think the informal communications was a strength that came from the relationship." [PAH Ex, 28] 
When RDH CMN were asked to provide an overall assessment of the nursing rotations these staff unanimously responded "very positive" (among response options; 'somewhat positive', 'neutral', 'somewhat negative' or 'very negative') $(\mathrm{n}=8)$. These staff members indicated that they wished the partnership could continue.

At the executive level at the NCCTRC and PAH, respondents also provided positive overall appraisals of the partnership, with the termination of the partnership noted to be due to a national change of strategic direction of the NCCTRC and not a reflection of the partnership.

"Broadly speaking the relationship has been an outstanding one, so the concept of actually partnering with PAH which is well known as a Magnet hospital was a very good one". [NCCTRC Ex, 1]

Many staff members spoke of the partnership as something that they were very grateful and proud to have been a part of and described numerous and lasting benefits for stakeholders.

"I'd say I'm proud to be a part of such a fantastic four years. I mean it was one of those things that you don't often get the opportunity to do and I think the value that our team have put into that hasn't just ceased with the end of a four-year contract. It's very much alive and well, right now, today in the Royal Darwin Hospital with the input from education and policies". [PAH Ex, 28]

"In summary the relationship has been an incredibly good one. It involved a significant growth in nursing maturity on this side, the RDH side and has increased motivation and enthusiasm". [NCCTRC Ex, 1]

\section{Discussion}

This evaluation considered multiple perspectives to identify the strengths and weaknesses of the nursing partnership in the event of a national disaster. Evaluation of this type is essential to ensuring that the community has a proficient disaster management plan ( $\mathrm{Su}$ et al., 2013). 
A key component of the partnership's success was the implementation of selection processes to recruit suitable DSRT members. Nurse characteristics included those who were both highly skilled and motivated in order for the team to quickly and effectively assume their roles. Since staff were highly motivated to be a part of the partnership they were particularly careful to integrate with staff in RDH to enhance working relationships. RDH staff spoke of DSRT members being very welcome due to their high skill level and ability to adapt and acculturate to the RDH. The motivation level of the DSRT members led to high retention in the program and a great sense of responsibility in terms of exiting the program when they were not realistically available for deployment.

Preparation was key. Following time at RDH, DSRT members reported feeling well prepared to be deployed to RDH and felt confident that they had the necessary skills and knowledge to be a DRS team member. Objectively measuring participants' competence would provide a more rigorous assessment than that attained by self-assessment (Djalali et al., 2014). Preparedness for a disaster is acknowledged as a key requisite for disaster services (Gorlin \& Hick, 2013; Hammad et al., 2012). Aspects of adequate preparation noted in the current study included on-site periods at RDH (rather than undergoing a virtual orientation), and receiving education and training about, equipment (including computers) and cultural matters. DSRT members indicated that they would have liked to have received more information and training with regard to caring for paediatric patients.

Most DSRT members indicated that the rotations had little or no negative impact on their families. This is consistent with what PAH DRS team members have reported in previous evaluations (Mitchell et al., 2014). Adequate family preparedness is known to influence whether nurses can be successfully deployed (Arbon et al., 2013). 
Communication was identified as an area of the partnership that was not always ideal. This was perhaps always likely to be an issue given that the hospitals were a great distance apart. Practical considerations were noted such as the need to plan well in advance at the hospital providing staff to ensure sufficient staff numbers and adequate skill mix among remaining staff. The fact that the DSRT members tried to fit in with the RDH and that the RDH acknowledged and appreciated their expertise is likely to have contributed to positive working relationships, which are known to improve work satisfaction (Lu et al., 2012; Sawatzky and Enns, 2012). The provision of adequate housing and transport for the DSRT when attending $\mathrm{RDH}$ was noted as important.

The partnership achieved its principle outcome in terms of having suitably prepared nurses ready to attend the RDH to provide 'back-fill' in the event of a disaster. Team members reported confidence in their skills and knowledge which was reflected in feedback provided by RDH senior staff. The disaster exercise confirmed that large numbers of team members were available when called on to be deployed. The availability rate of $79 \%$ demonstrated in the disaster exercise, is higher than the $50 \%$ availability rate observed among disaster response workers elsewhere (Zagelbaum et al., 2014). This difference was likely due to the unique purpose of the DRST, the recruitment procedures and ongoing rotations, and the commitment and ownership that the nurses felt towards the partnership.

Many positive benefits from the partnership were evident including: sharing of information (both within and between the partner entities), collegial relationship building, organisational relationship building, positive staff morale, and enhancement of organisational and national 
disaster preparedness. The cessation of the partnership means that many of the advances made in these areas are not likely to endure.

\section{Limitations}

During the four year partnership, no actual disaster occurred that required DSRT involvement. This limited the ability to fully trial the partnership in an authentic manner. The disaster exercise, although helpful in trialing the processes and assessing nurse availability, has some limitations.

\section{Conclusions}

This paper contributes to the small body of literature that addresses the practicalities of staffing supply as an important component of disaster preparedness. Specifically, this study sought to describe and evaluate a four-year Australian nursing partnership between two diverse and geographically distanced hospitals that aimed to provide a ready workforce in the event of national disasters. Obtaining feedback from the three partner entities (NCCTRC, $\mathrm{RDH}$ and $\mathrm{PAH}$ ) and including staff at various levels of involvement in the partnership (DSRT, senior nurses and executive staff) allowed a comprehensive examination of this disaster preparedness initiative.

The study revealed some practical considerations vital to the success and ongoing improvement of such organisational partnerships. These related to the selection and preparation of staff, structural and organisational policies regarding communication and staff rostering. The findings on which factors enhanced or hindered their participation and satisfaction with the program, and which ultimately influenced the program's success, are likely to be applicable to other disaster response nursing preparations conducted in western 
countries. The partnership met its principal goal of having a suitably prepared and available on-call nursing workforce to provide 'back-fill' to the host organisation in the event of a disaster. 
References

Albrito, P 2012, 'Making cities resilient: Increasing resilience to disasters at the local level', Journal of business continuity and emergency planning, vol. 5, no. 4, pp. 291-297. Annells, M \& Whitehead, D 2007, ‘Analysis data in qualitative research', in Z Schneider, D Whitehead, D Elliott, G Lobiondo-Wood, \& J Haber (eds.), Nursing \& Midwifery Research: Methods and Appraisal for Evidence-Based Practice, 3rd edn, Elsevier, Sydney.

Arbon, P, Ranse, J, Cusack, L, Considine, J, Shaban, R, Woodman, RJ, Banisch, L, Kako, M, Hammad, K \& Mitchell, B 2013, ‘Australasian emergency nurses’ willingness to attend work in a disaster: A survey', Australasian Emergency Nursing Journal, vol. 16, no. 2 , pp. $52-57$.

Arbon, P, Cusack, L, Ranse, J, Shaban, R, Considine, J, Kako, M, Woodman, RJ, Mitchell, B, Banisch, L \& Hammad, K 2013, 'Exploring staff willingness to attend work during a disaster: A study of nurses employed in four Australian emergency departments', Australasian Emergency Nursing Journal, vol. 16, no. 3, pp. 103-109.

Centre for Research on the Epidemiology of Disasters 2009, Natural Disaster Trends, CRED, viewed 10 February 2015, http://www.emdat.be/natural-disasters-trends

Chapman K \& Arbon P. 'Are nurses ready? Disaster preparedness in the acute setting', 2008 Australasian Emergency Nursing Journal, vol. 11, pp. 135-144.

Creswell, JW 2009, Research Design: Qualitative, Quantitative, and Mixed Methods Approaches, Sage Publications, Thousand Oaks, CA.

Department for International Development 2011, Humanitarian Emergency Response Review, viewed on 17 April 2015, https://www.gov.uk/government/uploads/system/uploads/attachment_data/file/67579/ HERR.pdf 
Djalali, A, Ingrassia, PL, Della Corte, F, Foletti, M, Ripoll Gallardo, A, Ragazzoni, L, Kaptan, K, Lupescu, O, Arculeo, C, von Arnim, G, Friedl, T, Ashkenazi, M, Heselmann, D, Hreckovski, B, Khorrram-Manesh, A, Komadina, R, Lechner, K, Patru, C, Burkle, FM Jr., \& Fisher, P 2014, 'Identifying deficiencies in national and foreign medical team responses through expert opinion surveys: implications for education and training', Prehospital and Disaster Medicine, vol. 29, no. 4, pp. 364368.

Duarte, V \& Haynes, LC 2006, 'Disaster preparedness: “as common as CRP”, Clinical Simulation in Nursing Education, vol 2, e53-e57.

Gorlin, JB \& Hick, JL 2013, 'Minneapolis bridges falling down: Emergency transfusion preparedness', Transfusion and Apheresis Science, vol. 49, pp. 403-407.

Hammad, KS, Arbon, P, Gebbie, K \& Hutton, A 2012, 'Nursing in the emergency department (ED) during a disaster: A review of the current literature', Australasian Emergency Nursing Journal, vol. 15, pp. 235-244.

International Federation of Red Cross and Red Crescent Societies 2001, World Disasters Report 2000, International Federation of Red Cross and Red Crescent Societies, Geneva.

Jennings-Sanders, A, Frisch, N \& Wing, S 2005, 'Nursing Students' Perceptions About Disaster Nursing', vol. 3, no.3, pp. 80-85.

Jones, A, and Bugge, C 2006, 'Improving understanding and rigour through triangulation: an exemplar based on patient participation in interaction', Journal of Advanced Nursing, vol. 55, no. 5, pp. 612-621.

Lesus, XR 2000, 'The Road Ahead', Prehospital and Disaster Medicine, vol. 15, no. 4, pp. 136-143. 
Lu, H, Barriball, KL, Zhang, X \& While, A 2012, 'Job satisfaction among hospital nurses revisited: A systematic review', International Journal of Nursing Studies, vol. 49, pp. 1017-1038.

Mitchell, ML, Mackie, B, Aitken, LM \& McKinnon, L 2014, 'Evaluation of an Australian nursing partnership to improve disaster response capacity', Disaster Prevention and Management, vol. 2, no. 5, pp. 524-532.

Pang, SMC, Chan, SSS \& Cheng, Y 2009, 'Pilot training program for developing disaster nursing competencies among undergraduate students in China', Nursing and Health Sciences, vol 11, pp. 367-373.

Pattillo, M 2006, 'Teaching disaster nursing response using simulations', Clinical Simulation in Nursing Education, vol. 2, e49-e51.

Redmond, AD 2005, 'Natural disasters', British Medical Journal, vol. 330, no. 7502, pp. $1259-1261$.

Sawatzky, JA \& Enns, CL 2012, 'Exploring the key predictors of retention in emergency nurses', Journal of Nursing Management, vol. 20, pp. 696-707.

Su, T, Han, X, Chen, F, Du, Y 2013, 'Knowledge levels and training needs of disaster medicine among health professionals, medical students, and local residents in Shanghai, China', PLoS ONE. vol.8, no.6:e67041, doi:10.1371/journal.pone.0067041.

Teddlie, C \& Tashakkori , A 2013, 'Major issues and controversies in the use of mixed methods in the social and behavioral sciences', in A Tashakkori, C Teddlie (eds.), Handbook of Mixed Methods in Social \& Behavioral Research, Sage Publications, Thousand Oakes, CA.

Zagelbaum, NK, Heslin, KC, Stein, JA, Ruzek, J, Smith, RE, Nyugen, T \& Dobalian, A 2014, 'Factors influencing readiness to deploy in disaster response: findings from a cross-sectional survey of the Department of Veterans Affairs Disaster Emergency 
Medical Personnel System', BMC Emergency Medicine, viewed on 10 December, 2014 http://www.biomedcentral.com/1471-227X/14/16. 
Table 1 Profile of Disaster Support Response Team $(n=38)$

\begin{tabular}{|c|c|c|c|}
\hline \multicolumn{2}{|l|}{ Characteristic } & \multirow{2}{*}{\multicolumn{2}{|c|}{$\begin{aligned} \mathrm{N} & (\%) \\
8 & (21)\end{aligned}$}} \\
\hline Gender & male & & \\
\hline & female & 30 & (79) \\
\hline \multirow[t]{5}{*}{ Age (years) } & $25-34$ & 20 & (53) \\
\hline & $35-44$ & 11 & (29) \\
\hline & $45-54$ & 4 & (11) \\
\hline & $55-64$ & 2 & (5) \\
\hline & missing & 1 & (3) \\
\hline \multirow{3}{*}{$\begin{array}{l}\text { Highest educational } \\
\text { attainment }\end{array}$} & Bachelor's Degree & 15 & (39) \\
\hline & Graduate Certificate & 19 & $(50)$ \\
\hline & Master's Degree & 4 & (11) \\
\hline \multirow[t]{4}{*}{ Number of rotations } & 1 & 18 & (47) \\
\hline & 2 & 10 & $(26)$ \\
\hline & 3 & 5 & (13) \\
\hline & $>3$ & 5 & (13) \\
\hline \multirow[t]{4}{*}{ Work area } & ICU & 10 & $(26)$ \\
\hline & ED & 11 & (29) \\
\hline & OT & 9 & (24) \\
\hline & PACU & 8 & $(21)$ \\
\hline
\end{tabular}

Abbreviations: ICU = Intensive Care Unit, ED = Emergency Department, OT= Operating Theatre, $\mathrm{PACU}=$ Perioperative and Anaesthetics. 
Table 2. Self-assessment by DSRT of their knowledge and skills related to their role $(n=38)$.

\begin{tabular}{lllll}
\hline \multicolumn{1}{c}{ Item } & \multicolumn{3}{c}{ Response } \\
\hline $\begin{array}{l}\text { I felt comfortable that I had the } \\
\text { knowledge and skills to: }\end{array}$ & $\begin{array}{c}\text { Strongly } \\
\text { agree } \\
\mathrm{n}(\%)\end{array}$ & $\begin{array}{c}\text { Agree } \\
\mathrm{n}(\%)\end{array}$ & $\begin{array}{c}\text { Disagree } \\
\mathrm{n}(\%)\end{array}$ & $\begin{array}{c}\text { Strongly } \\
\text { Disagree } \\
\mathrm{n}(\%)\end{array}$ \\
\hline $\begin{array}{c}\text { Perform my role, as a DRST } \\
\text { member }\end{array}$ & $24(63)$ & $13(34)$ & $0(0)$ & $0(0)$ \\
$\quad \begin{array}{l}\text { Understand expectations of my } \\
\text { performance as a member of DRST }\end{array}$ & $16(42)$ & $19(50)$ & $1(3)$ & $0(0)$ \\
$\begin{array}{l}\text { Understand RDH and PAH } \\
\text { leadership expectations of my clinical } \\
\text { performance }\end{array}$ & $18(47)$ & $17(45)$ & $1(3)$ & $0(0)$ \\
$\begin{array}{l}\text { Identify resources for my work on } \\
\text { the DRST }\end{array}$ & $11(29)$ & $26(68)$ & $0(0)$ & $0(0)$
\end{tabular}

${ }^{+}$Missing data $(\mathrm{n}=1),{ }^{\wedge}$ Missing data $(\mathrm{n}=2)$ Abbreviations: $\mathrm{RDH}=$ Royal Darwin Hospital, PAH = Princess Alexandra Hospital, DSRT $=$ Disaster Support Response Team. 
Table 3. Self-assessment by DSRT of their knowledge and skills in caring for Aboriginal and Torres Strait Islander patients and their families $(n=38)$.

\begin{tabular}{|c|c|c|c|c|}
\hline Item & \multicolumn{4}{|c|}{ Response } \\
\hline $\begin{array}{l}\text { I felt comfortable that I had the knowledge } \\
\text { and skills to: }\end{array}$ & $\begin{array}{l}\text { Strongly } \\
\text { agree } \\
\mathrm{n}(\%)\end{array}$ & $\begin{array}{l}\text { Agree } \\
\mathrm{n}(\%)\end{array}$ & $\begin{array}{l}\text { Disagree } \\
\mathrm{n}(\%)\end{array}$ & $\begin{array}{c}\text { Strongly } \\
\text { Disagree } \\
\mathrm{n}(\%)\end{array}$ \\
\hline $\begin{array}{l}\text { Understand expectations of my } \\
\text { performance in relation to caring for } \\
\text { Aboriginal and Torres Strait Islander } \\
\text { patients and their families }\end{array}$ & $9(24)$ & $25(66)$ & $2(5)$ & $0(0)$ \\
\hline $\begin{array}{l}\text { Perform my role caring for Aboriginal } \\
\text { and Torres Strait Islander patients and their } \\
\text { families }^{+}\end{array}$ & $6(16)$ & $29(76)$ & $2(5)$ & $0(0)$ \\
\hline $\begin{array}{l}\text { Identify resources for my work in } \\
\text { caring for Aboriginal and Torres Strait } \\
\text { Islander patients and their families }{ }^{\wedge}\end{array}$ & $8(21)$ & $22(58)$ & $6(16)$ & $0(0)$ \\
\hline
\end{tabular}


Table 4. Agreement by DSRT regarding working relationships with RDH staff ( $n=38)$.

\begin{tabular}{lcccc}
\hline \multicolumn{1}{c}{ Item } & \multicolumn{3}{c}{ Response } \\
\hline Working relationships & $\begin{array}{c}\text { Strongly } \\
\text { agree } \\
\mathrm{n}(\%)\end{array}$ & $\begin{array}{l}\text { Agree } \\
\mathrm{n}(\%)\end{array}$ & $\begin{array}{c}\text { Disagree } \\
\mathrm{n}(\%)\end{array}$ & $\begin{array}{c}\text { Strongly } \\
\text { Disagree } \\
\mathrm{n}(\%)\end{array}$ \\
\hline $\begin{array}{l}\text { I felt comfortable that I had the } \\
\text { knowledge and skills to develop effective } \\
\text { working relationships with unit staff }\end{array}$ & $20(53)$ & $16(42)$ & $1 \quad(3)$ & $0(0)$ \\
$\quad \begin{array}{l}\text { I felt accepted by my RDH co-workers } \\
\text { as a member of the team }\end{array}$ & $10(26)$ & $26(68)$ & 1 (3) & $0(0)$ \\
$\quad \begin{array}{l}\text { I felt accepted by the RDH } \\
\text { multidisciplinary team }\end{array}$ & $13(34)$ & $19(50)$ & $5(13)$ & $0(0)$
\end{tabular}

${ }^{\wedge}$ Missing data $(\mathrm{n}=1)$. Abbreviations: DSRT = Disaster Support response Team; RDH = Royal Darwin Hospital. 
Table 5. Rostering implications for PAH and RDH.

\begin{tabular}{llll}
\hline & Impact & PAH $(\mathrm{n}=10)$ & RDH $(\mathrm{n}=9)$ \\
\hline Staff rostering & No impact & 0 & 5 \\
& Some impact & 4 & 1 \\
& Moderate impact & 3 & 1 \\
& Large impact & 2 & 2 \\
N/A & 1 & 0
\end{tabular}

Abbreviations: RDH = Royal Darwin Hospital, PAH = Princess Alexandra Hospital. 